\title{
A concavity property for the measure of product sets in groups
}

\author{
by
}

\author{
Imre Z. Ruzsa* (Budapest)
}

\begin{abstract}
Let $G$ be a connected locally compact group with a left invariant Haar measure $\mu$. We prove that the function $\xi(x)=\inf \{\bar{\mu}(A B): \mu(A)=x\}$ is concave for any fixed bounded set $B \subset G$. This is used to give a new proof of Kemperman's inequality $\underline{\mu}(A B) \geq \min (\underline{\mu}(A)+\underline{\mu}(B), \mu(G))$ for unimodular $G$.
\end{abstract}

1. Introduction. Let $G$ be a locally compact topological group, and let $\mu$ denote a left invariant Haar measure, that is, one satisfying $\mu(x A)=\mu(A)$ for every $x \in G$ and $A \subset G$. We use $\underline{\mu}, \bar{\mu}$ to denote the corresponding inner and outer measures.

It is well known that there is a continuous homomorphism $\Delta: G \rightarrow$ $\left(\mathbb{R}^{+}, \cdot\right)$ such that

$$
\mu(A x)=\Delta(x) \mu(A)
$$

for all $x$ and $A$. $\Delta$ is called the modular function of the group. If $\Delta(x) \equiv 1$, that is, the left Haar measure is also right invariant, $G$ is called unimodular.

We call a set bounded if its closure is compact.

1.1. Definition. For a fixed bounded set $B \subset G$, we define its impact function $\xi=\xi_{B}$ for $x>0$ by

$$
\xi(x)=\inf \{\bar{\mu}(A B): \mu(A)=x\} .
$$

This function is defined for all values of $x>0$ that can serve as a value of $\mu(A)$. If $G$ is not discrete, $\mu$ is not atomic, and then this means all $0<x \leq \mu(G)$. In this case for all $0<y<x$, every set of measure $x$ has a subset of measure $y$, which shows that $\xi$ is monotonically increasing (not necessarily strictly, as easy examples show).

* Supported by Hungarian National Foundation for Scientific Research, Grant No. 1901. 
At $x=0$ we define $\xi(0)$ by continuity. Definition (1.2) would yield 0 , and additionally requiring $A \neq \emptyset$ we would obtain $\bar{\mu}(B)$. The limit satisfies

$$
\bar{\mu}(B) \leq \xi(0) \leq \mu(\bar{B}),
$$

where $\bar{B}$ is the closure of $B$. For the additive group of reals it is possible to show that there is equality on the right.

The term "impact function" comes from additive number theory. Plünnecke (1969) uses the term "Wirkungsfunktion" for a similar quantity related to the density of sums of sets of integers, of which "impact function" is a literal translation.

Our main result is the following.

THEOREM 1. If $G$ does not have any proper compact-open subgroup, then $\xi$ is a continuous concave function on its whole domain.

This will be applied to obtain the following corollaries.

THEOREM 2. If $G$ is a compact, connected group, then for arbitrary $A, B \subset G$ we have

$$
\underline{\mu}(A B) \geq \min (\underline{\mu}(A)+\underline{\mu}(B), \mu(G)) .
$$

THEOREM 3. If $G$ is noncompact and does not have any proper compactopen subgroup, then for arbitrary $A, B \subset G$ we have

$$
\underline{\mu}(A B) \geq \underline{\mu}(A) \sup _{b \in B} \Delta(b)+\underline{\mu}(B) .
$$

If $G$ is unimodular, then (1.4) reduces to the simple form

$$
\underline{\mu}(A B) \geq \underline{\mu}(A)+\underline{\mu}(B) .
$$

(1.3) and (1.5) are due to Kemperman (1964). Important special cases were established earlier: see Raikov (1939) for the case of the circle, Macbeath (1953) for the $n$-dimensional torus, Shields (1955) for connected commutative compact second countable groups, Kneser (1956) for commutative locally compact groups. Kemperman describes the structure of sets that do not satisfy (1.3) or (1.5) if the group has compact-open subgroups; this does not seem to be easily available by the method of this paper.

For non-unimodular $G$ Kemperman shows that (1.5) fails, and he gives the following inequality:

$$
\frac{\underline{\mu}(B)}{\underline{\mu}(A B)}+\frac{\underline{\nu}(A)}{\underline{\nu}(A B)} \leq 1,
$$

where $\nu$ is a right Haar measure. In Section 4 we show that (1.6) follows from (1.4). A proof of (1.4) with Kemperman's method of proving (1.6) is also possible. 
2. Proof of the concavity. We always assume that $G$ does not have any proper compact-open subgroup. By well-known results about the component structure of locally compact groups (see Hewitt-Ross (1963), Chapter 2, Section 7), for compact $G$ this means that $G$ is connected, and for noncompact $G$ it means that the component of unity is unbounded. of

2.1. Lemma. Let $A \subset G, 0<\mu(A)=x<\infty$. The set of possible values

$$
\mu(A \cap(g A)), \quad g \in G,
$$

contains the interval $(x-u, x]$, where $u=x(1-x / \mu(G))$ if $G$ is compact, and $u=x$ otherwise.

Proof. (2.1) is a continuous function of $g$ and its value at the unity is $x$. If $G$ is compact, then a simple application of Fubini's theorem reveals that

$$
\int_{G} \mu(A \cap(g A)) d \mu(g)=\mu(A)^{2}=x^{2},
$$

hence it can be as small as $x^{2} / \mu(G)=x-x(1-x / \mu(G))$. By the connectedness of $G$, every value between the established lower limits and $x$ is also attained by the function (2.1).

If $G$ is not compact, we consider (2.1) only on the component of unity. It can be arbitrarily small; in fact, it will be $<\varepsilon$ outside a compact set depending on $A$ and $\varepsilon$. Consequently, by continuity it attains every value in $(0, x]$.

2.2. Lemma. For all $X, Y \subset G$ we have

$$
\bar{\mu}(X \cap Y)+\bar{\mu}(X \cup Y) \leq \bar{\mu}(X)+\bar{\mu}(Y)
$$

Proof. Take measurable sets $X^{\prime}, Y^{\prime}$ such that $X \subset X^{\prime}, Y \subset Y^{\prime}$, $\mu\left(X^{\prime}\right)=\bar{\mu}(X), \mu\left(Y^{\prime}\right)=\bar{\mu}(Y)$. (2.2) follows from the equality

$$
\mu\left(X^{\prime} \cap Y^{\prime}\right)+\mu\left(X^{\prime} \cup Y^{\prime}\right)=\mu\left(X^{\prime}\right)+\mu\left(Y^{\prime}\right)
$$

and the obvious inclusions.

Proof of Theorem 1. First we establish the "local concavity" of the function $\xi$ in the following sense. Take an $x>0(x<\mu(G)$ if $G$ is compact). We show that

$$
\xi(x+h)+\xi(x-h) \leq 2 \xi(x)
$$

for $h<x(1-x / \mu(G))$ (compact case), or for $h<x$ (noncompact case).

Take an $\varepsilon>0$ and a set $A$ such that

$$
\mu(A)=x, \quad \bar{\mu}(A B)<\xi(x)+\varepsilon .
$$

Take a $g \in G$ such that $\mu(A \cap(g A))=x-h$. Such a $g$ exists by Lemma 1 , and it also satisfies $\mu(A \cup(g A))=x+h$ by (2.3). Applying Lemma 2 for 
the sets $X=A B, Y=g A B$ we find

$$
\begin{aligned}
\bar{\mu}((A B) \cap(g A B))+\bar{\mu}((A B) \cup(g A B)) & \leq \bar{\mu}(A B)+\bar{\mu}(g A B) \\
& =2 \bar{\mu}(A B)<2 \xi(x)+2 \varepsilon .
\end{aligned}
$$

We also know that $(A B) \cap(g A B) \supset(A \cap(g A)) B$, hence $\bar{\mu}((A B) \cap(g A B)) \geq$ $\bar{\mu}((A \cap(g A)) B)) \geq \xi(x-h)$, and $(A B) \cup(g A B)=(A \cup(g A)) B$, so $\bar{\mu}((A B) \cup$ $(g A B)) \geq \bar{\mu}((A \cup(g A)) B)) \geq \xi(x+h)$. These inequalities together yield that

$$
\xi(x-h)+\xi(x+h) \leq 2 \xi(x)+\varepsilon ;
$$

since this holds for every $\varepsilon>0,(2.4)$ is established.

The function $\xi$ is obviously bounded on every finite interval, hence it is continuous and concave on every interval in which (2.4) holds. In the noncompact case (2.4) is restricted only by the obvious $h<x$, thus concavity on the whole line $(0, \infty)$ follows. In the compact case we can conclude concavity on every interval $(a, b)$ where $b^{2} / \mu(G)<a<b$. Now if a function is continuous and concave on two overlapping intervals, it is so in their union as well. Since any two points $0<u<v<\mu(G)$ can be connected by a finite chain of overlapping intervals of this type, we infer concavity on the whole $(0, \mu(G))$.

We can also add the endpoints. At the point 0 , continuity follows by the definition, and at $\mu(G)$ (compact case) the obvious inequalities $x \leq \xi(x) \leq$ $\mu(G)$ imply continuity. Hence the concavity also extends to these points.

\section{Properties of the impact function}

3.1. Proposition. Assume that $G$ is compact and connected. Write $B^{-1}=B^{\prime}, \xi(x)=\xi_{B}(X), \xi^{\prime}(x)=\xi_{B^{\prime}}(x)$. The smallest value of $x$ for which $\xi(x)=\mu(G)$ is $x=\mu(G)-\xi^{\prime}(0)$, and the smallest value of $x$ for which $\xi^{\prime}(x)=\mu(G)$ is $x=\mu(G)-\xi(0)$. The graph of $\xi(x)$ on the interval $\left[0, \mu(G)-\xi^{\prime}(0)\right]$ and the graph of $\xi^{\prime}(x)$ on the interval $[0, \mu(G)-\xi(0)]$ are reflections of each other about the line $x+y=\mu(G)$.

Proof. Consider the hypergraphs of $\xi$ and $\xi^{\prime}$, that is, the set

$$
S=\{(x, y): 0<x, y<\mu(G), y>\xi(x)\}
$$

and the analogous set $S^{\prime}$ for $\xi^{\prime}$. We show that if $(x, y) \in S$, then $(\mu(G)-$ $y, \mu(G)-x) \in S^{\prime}$. Indeed, take $A \subset G$ such that $\mu(A)>x, \bar{\mu}(A B)<y$. (We can take a set of measure $>x$ rather than exactly $x$ by the continuity of $\xi$.) We have

$$
\underline{\mu}(G \backslash A B)=\mu(G)-\bar{\mu}(A B)>\mu(G)-y .
$$

Take $A^{\prime} \subset G \backslash A B$ for which $\mu\left(A^{\prime}\right)=\mu(G)-y$. Since $A B \cap A^{\prime}=\emptyset$, we have 
$A \cap A^{\prime} B^{\prime}=\emptyset$. This implies that

$$
\bar{\mu}\left(A^{\prime} B^{\prime}\right) \leq \mu(G)-\mu(A)<\mu(G)-x,
$$

hence $\xi^{\prime}(\mu(G)-y)<\mu(G)-x$ as wanted.

We have shown that the hypergraphs $S$ and $S^{\prime}$ are reflections of each other, which immediately implies the reflection property of the graphs on the corresponding open intervals. This can be extended to the closed intervals by continuity, and this implies the statements concerning the points where $\xi$ and $\xi^{\prime}$ reach $\mu(G)$.

This property also has an analog in additive number theory (see Plünnecke (1969), Chapter 7).

3.2. Corollary. Assume that $G$ is compact, commutative and connected. The smallest value of $x$ for which $\xi(x)=\mu(G)$ is $x=\mu(G)-\xi(0)$. The graph of $\xi(x)$ on the interval $[0, \mu(G)-\xi(0)]$ is symmetric about the line $x+y=\mu(G)$.

Proof. Since $\mu(A B)=\mu\left(A^{-1} B^{-1}\right)$, we have $\xi=\xi^{\prime}$.

3.3. Corollary. With $c=\xi(0), c^{\prime}=\xi^{\prime}(0)$ we have

$$
\xi(x) \geq \min \left(c+\frac{\mu(G)-c}{\mu(G)-c^{\prime}} x, \mu(G)\right)
$$

for all $x$.

Proof. This is a consequence of $\xi(0)=c, \xi\left(\mu(G)-c^{\prime}\right)=\mu(G)$ and the concavity.

3.4. Corollary. In a compact connected group we have

$$
\xi(x) \geq \min (x+\bar{\mu}(B), \mu(G)) .
$$

Proof. This follows from (3.1) and $\xi(0) \geq \bar{\mu}(B), \xi^{\prime}(0) \geq \bar{\mu}\left(B^{-1}\right)=$ $\bar{\mu}(B)$.

3.5. Proposition. Assume that $G$ is noncompact and does not have any proper compact-open subgroup. The function

$$
\xi(x)-x \sup _{b \in B} \Delta(b)
$$

is increasing, and we have

$$
\xi(x) \geq x \sup _{b \in B} \Delta(b)+\xi(0) .
$$

Pro of. Take an arbitrary $b \in B$. For any measurable set $A$ we have

$$
\bar{\mu}(A B) \geq \mu(A b)=\Delta(b) \mu(A),
$$

hence $\xi(x) \geq \Delta(b) x$. Taking the supremum we obtain

$$
\xi(x) \geq x \sup _{b \in B} \Delta(b) .
$$


This means that the function in (3.3) is nonnegative. Since it is concave, it must be increasing. In (3.4) we just compare its values at $x$ and 0 .

3.6. COROLlary. Under the same assumptions we have

$$
\xi(x) \geq x \sup _{b \in B} \Delta(b)+\bar{\mu}(B) .
$$

Proof. This follows from $(3.4)$ and $\xi(0) \geq \bar{\mu}(B)$.

\section{Inequalities for the measure of product sets}

4.1. Proposition. In a compact connected group for arbitrary nonempty $A, B \subset G$ we have

$$
\bar{\mu}(A B) \geq \min (\underline{\mu}(A)+\bar{\mu}(B), \mu(G)) .
$$

Proof. If $\mu(A)=0,(4.1)$ is obvious. If $\underline{\mu}(A)>0$, take a measurable $A^{\prime} \subset A$ with $\mu \overline{\left(A^{\prime}\right)}=\mu(A)$. We have

$$
\bar{\mu}(A B) \geq \bar{\mu}\left(A^{\prime} B\right) \geq \xi(\underline{\mu}(A))
$$

and an application of (3.2) completes the proof.

Proof of Theorem 2. Take an $\varepsilon>0$ and compact sets $A^{\prime} \subset A$, $B^{\prime} \subset B$ with $\mu\left(A^{\prime}\right)>\underline{\mu}(A)-\varepsilon, \mu\left(B^{\prime}\right)>\underline{\mu}(B)-\varepsilon$. We have

$$
\underline{\mu}(A B) \geq \underline{\mu}\left(A^{\prime} B^{\prime}\right)=\bar{\mu}\left(A^{\prime} B^{\prime}\right) \geq \min (\underline{\mu}(A)+\underline{\mu}(B)-2 \varepsilon, \mu(G))
$$

by (4.1). This holds for every positive $\varepsilon$, which implies (1.3).

4.2. Proposition. Assume that $G$ is noncompact and does not have any proper compact-open subgroup. For arbitrary nonempty $A, B \subset G$ we have

$$
\bar{\mu}(A B) \geq \underline{\mu}(A) \sup _{b \in B} \Delta(b)+\bar{\mu}(B) .
$$

Proof. This follows from (3.5) in the same way as the previous proposition was deduced from (3.2).

Proof of Theorem 3. Same as that of Theorem 2, applying (4.2) in place of (4.1).

Finally, we show how Theorem 3 implies (1.6) for non-unimodular groups.

Proof of (1.6). We may assume that $\nu$ is the right Haar measure associated with $\mu$, that is, we have

$$
\nu(X)=\mu\left(X^{-1}\right)
$$

and

$$
\mu(X)=\int_{X} \Delta d \nu
$$

for every measurable set $X$. 
Assume now $\mu(B)>0$ (otherwise (1.6) does not make sense). Taking a measurable $B^{\prime} \subset \bar{B}$ with $\mu\left(B^{\prime}\right)=\underline{\mu}(B)$ we find

$$
\underline{\mu}(B)=\mu\left(B^{\prime}\right)=\int_{B^{\prime}} \Delta d \nu \leq \nu\left(B^{\prime}\right) \sup _{b \in B} \Delta(b) \leq \underline{\nu}(B) \sup _{b \in B} \Delta(b) .
$$

Hence (1.4) implies that

$$
\underline{\mu}(A B) \geq \underline{\mu}(A) \frac{\underline{\mu}(B)}{\underline{\nu}(B)}+\underline{\mu}(B),
$$

which can be rearranged as

$$
\frac{\underline{\mu}(B)}{\underline{\mu}(A B)} \leq \frac{\underline{\nu}(B)}{\underline{\mu}(A)+\underline{\nu}(B)} .
$$

Applying (4.3) repeatedly from (4.4) we can deduce

$$
\frac{\underline{\nu}(A)}{\underline{\nu}(A B)} \leq \frac{\underline{\nu}\left(A^{-1}\right)}{\underline{\mu}\left(B^{-1}\right)+\underline{\nu}\left(A^{-1}\right)} \leq \frac{\underline{\mu}(A)}{\underline{\mu}(A)+\underline{\nu}(B)} .
$$

Adding (4.4) and (4.5) we obtain (1.6).

5. Problems. Kemperman (1964) also has a result for certain semigroups. Is a generalization of the concavity theorem to semigroups possible?

Assume that the component of unity is a compact-open semigroup $H$ with $\mu(H)=r$. Is it true that the function $\xi$ is concave in every interval $(k r,(k+1) r)$, where $0 \leq k<\mu(G) / \mu(H)$ is an integer?

Does the value of $\xi(x)$ change if in the definition (1.3) we require $A$ to be open, or bounded, or both? In which cases is the infimum a minimum?

Is it true that $\xi_{B}=\xi_{\bar{B}}$ for every set $B$ ? Is $\xi(0)$ equal to $\mu(\bar{B})$ ? An affirmative answer to the first question would naturally imply the same for the second.

If my conjecture $\xi(0)=\mu(\bar{B})$ holds, then (3.1) and (3.4) yield the following improvements of (3.2) and (3.5):

$$
\xi(x) \geq \min (x+\mu(\bar{B}), \mu(G))
$$

in the compact case,

$$
\xi(x) \geq x \sup _{b \in B} \Delta(b)+\mu(\bar{B})
$$

in the noncompact case.

Also, in (4.1) and (4.2) the $\bar{\mu}(B)$ could be changed to $\mu(\bar{B})$ whenever $\mu(A)>0$. This also would yield improvements of Theorems 2 and 3 , namely on the left sides $\mu(A B)$ could be changed to $\mu(\operatorname{int} A B)$. This can be shown by using the same method as in the proof of the reflection property in Proposition 3.1. 


\section{References}

E. Hewitt and K. A. Ross, Abstract Harmonic Analysis, Springer, New York 1963.

J. H. B. Kemperman, On products of sets in a locally compact group, Fund. Math. 56 (1964), 51-68.

M. Kneser, Summenmengen in lokalkompakten abelschen Gruppen, Math. Z. 66 (1956), $88-110$.

A. M. Macbeath, On measure of sum sets II. The sum-theorem for the torus, Proc. Cambridge Philos. Soc. 49 (1953), 40-43.

H. Plünnecke, Eigenschaften und Abschätzungen von Wirkungsfunktionen, Ges. Mathematik und Datenverarbeitung, Bonn 1969.

D. A. Raikov, On the addition of point sets in the sense of Schnirelmann, Mat. Sb. 5 (47) (1939), 425-440 (in Russian).

A. Shields, Sur la mesure d'une somme vectorielle, Fund. Math. 42 (1955), 57-60.

MATHEMATICAL INSTITUTE

HUNGARIAN ACADEMY OF SCIENCES

BUDAPEST, PF. 127

H-1364 HUNGARY 